\title{
The Analysis on the Driving Error of the Servo Mechanism with a Harmonic Reducer
}

\author{
Xiang LI \\ Department of Electrical Engineering, Guangxi Technological College of Machinery and Electricity, \\ Nanning, 530007, China \\ email: xiangzi21th@hotmail.com
}

Keywords: Harmonic Drive, Servo Mechanism, Driving Error

\begin{abstract}
A harmonic reducer is a kind of gear transmission with high performance, as the servo mechanism with a harmonic reducer is widely used in the fields such as aviation and aerospace. For meeting the more higher precision of the servo mechanism, according to the operation principle and the configuration of the servo mechanism with a harmonic reducer, the driving errors of a harmonic reducer is analyzed and the error model is established. Moreover, the frequency formulas of the driving errors based on analyzing its mechanism and measuring results of dynamic performance is achieved. Finally, each frequency formula has been calculated with MATLAB and it is drawn a conclusion that it can be the basis to develop the process control of the actuator and enhance the reliability of actuators.
\end{abstract}

\section{Introduction}

Servo mechanism is the implementation parts of automatic control system [1], and is widely used in aerospace, aviation, medical equipment, machine tools, robots, etc [2]. Harmonic reducer, which is a kind of the device to realize the power transmission based on the elastic deformation of flexible middle components, is adopted as the main transmission mechanism in the servo mechanism with a harmonic reducer [3]. Compared to other forms of mechanical drive, harmonic drive has significant advantages [4]. So it is often used in the limited space and quality of Mechatronics systems, especially used in precision positioning equipment [5].

However, if the driving error of the servo mechanism with a harmonic reducer is too big, it is likely to cause system oscillation such as declining the servo accuracy or disturbing the servo system and its control circuit. On the other hand, if the clearance of a harmonic reducer is too small, it will have a giant effect on transmission efficiency and service life of servo mechanism such as its start torque and dynamic characteristic that it is likely to cause friction or damping increased greatly under the influence of various environmental conditions and load conditions, or even cause servo mechanism rest abnormally [6]. Thus it is necessary to research and analysis the driving error of the servo mechanism with a harmonic reducer in-depth.

\section{Ideal Transmission Model}

The servo mechanism with a harmonic reducer was composed of motor, cone gear decelerator, harmonic reducer, feedback device and shell, as show in figure 1, while circular flexible gear is used for the harmonic reducer. Its transmission principle as follow: it is driven by motor that the bevel pinion forms the first decelerator (Transmission Ratio $N_{1}$ ), and drive axis is rotated to $90^{\circ}$, then it is driven by the big bevel gear, while it is fitted together with wave generator of the harmonic reducer, of cone gear decelerator that the harmonic reducer forms the secondary decelerator (Transmission Ratio $N_{2}$ ), and the output is obtained through output rigid gear fitted together with flexible gear of the harmonic reducer [7].

Suppose that the motor is input, rigid gear is fixed, flexible gear is output, the first Transmission Ratio is formulated as $N_{1}=3$, the efficiency is $\eta_{1}$, the secondary Transmission Ratio is formulated as $N_{2}=100$, the efficiency is $\eta_{2}$, then 


$$
\begin{aligned}
& \theta_{f s}=-N_{2} \cdot \theta_{\mathrm{wg}}=N_{1} N_{2} \cdot \theta_{\mathrm{mt}} \\
& \omega_{f \mathrm{~s}}=-N_{2} \cdot \omega_{\mathrm{wg}}=N_{1} N_{2} \cdot \omega_{\mathrm{mt}}
\end{aligned}
$$

Assume that the other nonlinear factors is ignored, then the relation of torque of each transmission part is

$$
T_{\text {fs }}=\frac{1}{N_{2}+1} \cdot \eta_{2} T_{\mathrm{cs}}=-\frac{1}{N_{2}} \cdot \eta_{2} T_{\mathrm{wg}}=\frac{1}{N_{1} N_{2}} \cdot \eta_{1} \eta_{2} T_{\mathrm{mt}}
$$

In the type: $\theta_{m t} 、 \omega_{m t}$ and $T_{m t}$ respectively is angle, speed and the output torque of the motor; $\theta_{f s} 、 \omega_{f s}$ and $T_{f s}$ respectively is angle, speed and the load torque of the flexible gear; $\theta_{w g} 、 \omega_{w g}$ and $T_{w g}$ respectively is angle, speed and the load torque of the wave generator; and $T_{c s}$ is the load torque of the rigid gear [8] [9].

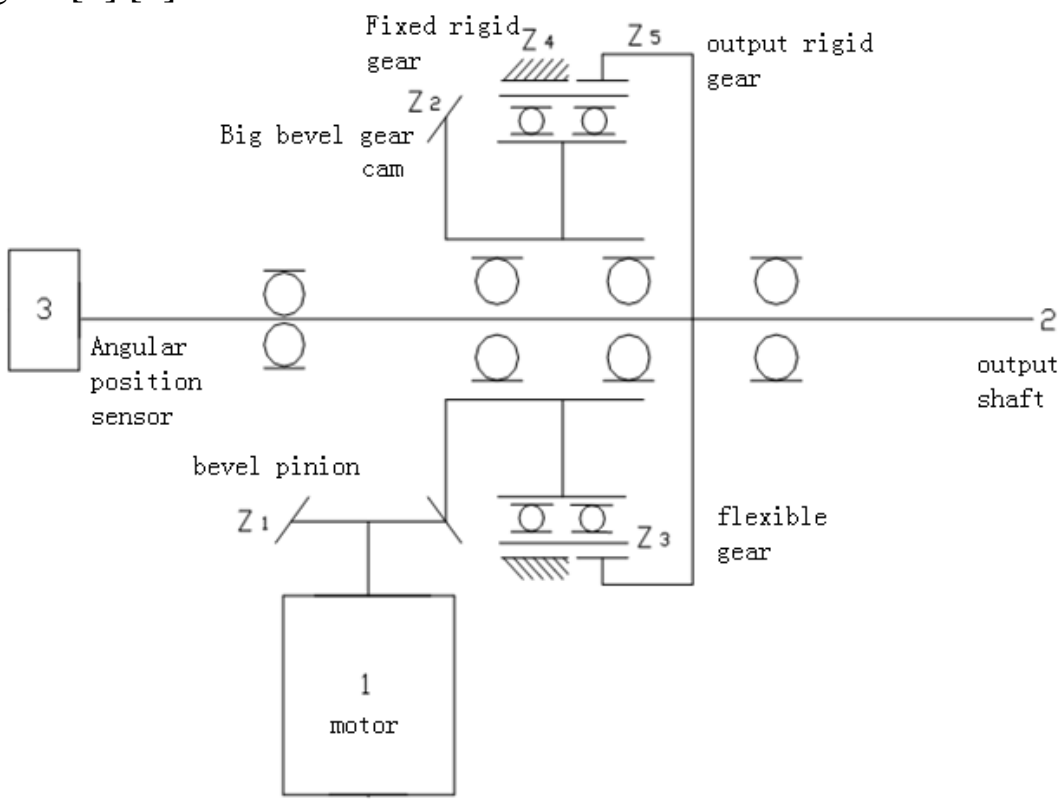

Fig. 1 the component diagram of servo mechanism with a harmonic reducer

\section{The Kinematics for Harmonic Reducer}

The principle of Harmonic gear drive is to realize the movement and power transmission relying on the continuous motion of the wave produced by which the wave generator force flexibility component (flexible gear) deformation. When the cylindrical flexible gear is working, the average integral angular velocity of its any point on the midline of each cross section is equal to the angular velocity of its no-deformation end based on the theorem of the average integrals angular velocity. Therefore, when the flexible gear stay in relative motion to the surface of a fixed wave generator, the shaft connected to flexible gear will keep turn with a constant angular velocity, so movement of the flexible gear can be simplified into rotary motion of rigid axis at a constant speed.

For this purpose, Willis methods, or so-called transformation mechanism, is usually used to analyze the harmonic reducer as shown in figure 1. Suppose that $\omega_{H}$ is a absolute angular velocity of the wave generator (Z2); $\omega_{1}$ is a absolute angular velocity of the flexible gear (Z3), or the equivalent average angular velocity or integral angular velocity of the deformation end of the flexible gear; $\omega_{2}$ is a absolute angular velocity of the fixed rigid gear (Z4).

According to the principle of relative motion[9], if add an angular velocity and wave generator to the gear train is equal and opposite direction to the size of the angular velocity $\left(-\omega_{H}\right)$, the relative movement of the relationship between each component remain unchanged. But for the entire gear train with a $\left(-\omega_{H}\right)$, it can be considered as stationary wave generator. So, the harmonic gear drive can be converted into is similar to the general internal gear transmission. In the transformation of 
institutions, soft and just wheel angular velocity of the relative wave generator are:

$$
\begin{gathered}
\omega_{1 H}=\omega_{1}-\omega_{H} \\
\omega_{2 H}=\omega_{2}-\omega_{H}
\end{gathered}
$$

If gear ratio of the flexible gear and the rigid gear is $u=z_{2} / z_{1}$, where $z_{1}, z_{2}$ respectively is the number of teeth on the flexible gear and the rigid gear, the drive ratio of conversion mechanism is

$$
i_{12}^{(H)}=\frac{\omega_{1 H}}{\omega_{2 H}}=\frac{\omega_{1}-\omega_{H}}{\omega_{2}-\omega_{H}}=u
$$

Due to the harmonic reducer of servo adopts double wave transmission, wave generator for CAM and to active parts, the flexible gear is the output, the fixed rigid gear is still, namely $\omega_{2}=0$. Therefore, according to equation (6) can be obtained

$$
i=\frac{\omega_{H}}{\omega_{1}}=\frac{1}{1-u}=-\frac{z_{1}}{z_{2}-z_{1}}
$$

If the harmonic reducer of servo in the flexible gear is $z_{1}=200$, and the rigid gear is $z_{2}=202$, so gear ratio of the harmonic reducer is $i=100$, reverse direction.

\section{Motion Error Model}

The composition and working principle known, the main error sources servos are: input angle error, the first stage reducer Backlash gap, the first stage reducer motion error, the second-stage gear gap and hysteresis, the second stage reduction implement motion error, transmission error and the stiffness caused by external load torque, etc., where the harmonic drive is the main source of error.

Harmonic gear reducer motion transmission error is due to the process of flexible wheel, just round intermeshing gear cumulative pitch error, and motion error generated in the engagement process. Motion error error sources are mainly radial dimension error generator radial motion error just wheel motion errors flexible wheel and an output shaft runout. While indexing circular error profile error in the harmonic drive cam, just round and soft wheel, profile error, error Flexspline elastic deformation produced uneven harmonic reducer that affect motion error, but eventually by affecting gear cumulative pitch error on motion error, it is similar to ordinary gear, so you can refer to ordinary gear motion error model to study the harmonic motion errors motion error model. Since the servo mechanism, the entire harmonic reducer support bearing assembly by a cam on the output shaft and the output shaft support bearing assembly through the housing to the housing, and therefore the need to consider the error model in the bearing clearance due to the assembly, the front and rear shell installation errors caused by precision body effect on the output error.

Since the harmonic drive engagement number, thus compensating for the error characteristics, if all of the error involved in tooth engagement are within the maximum tolerance range, the harmonic drive RMSE equal to ordinary Gear error multiplied by $k_{B} / \sqrt{z_{m p}}$, i.e.,

$$
\Delta \varphi_{h g}=\Delta \varphi_{g} \frac{k_{B}}{\sqrt{z_{m p}}}
$$

In the type: $\Delta \varphi_{h g}$ is Harmonic Drive motion error; $\Delta \varphi_{g}$ is Ordinary gear motion error; $z_{m p}$ is Harmonic gear meshing simultaneously involved in the actual logarithm; $k_{B}$ is Factor and is equal to the ratio of the measured and calculated values of motion errors, $k_{B}=0.8 \sim 1.0 . \Delta \varphi_{g}$ can be calculated by

$$
\Delta \varphi_{g}=\left[0.2 \sum_{1}^{j} \Delta F_{\Sigma j} i_{g}+0.3 \sqrt{\sum_{1}^{j}\left(\Delta F_{\Sigma j}\right)^{2}}\right] \frac{412.8}{d_{2}^{\prime}}
$$

In the type: $\Delta F_{\Sigma j}$ is comprehensive error for each gear $(\mu m) ; i_{g}$ is gear transmission ratio; $d_{2}^{\prime}$ 
is pitch circle diameter of the driven gear $(\mathrm{mm})$.

Compared with ordinary gear, because the harmonic drive more than a wave generator (cam) this additional link, which will inevitably affect the manufacturing error motion precision cam drive. Thus the harmonic drive, you must also equation (9) is included in the manufacturing errors of the cam. If this error is converted to an output shaft, they should be divided by the manufacturing error of the cam gear drive harmonic gear ratio $i_{h}$, while taking into account manufacturing error in the radial direction of the cam is given for the radial error equivalent up to the circumferential direction, tolerance should be multiplied by the radial direction of the cam angle liter contour tangent. If you take the average lead angle tooth contact area as calculated by the lead angle, the equivalent to the circumference of the output shaft of error:

$$
\Delta F_{g}=\frac{\pi r_{m}}{4 \omega_{0} i_{h}} \sum_{1}^{n} \Delta \rho_{n}
$$

In the type: $\Delta F_{g}$ is motion Error converted to the output shaft of the cam caused by a manufacturing error $(\mu \mathrm{m}) ; \Delta \rho_{n}$ is radial cam manufacturing errors $(\mu m) ; i_{h}$ is harmonic gear ratios; $r_{m}$ is the flexible gear diameter.

Just because of the difference between the number of teeth in the flexible gear and the rigid gear of harmonic drive is equal to the wave number, servo is dual wave transmission, wave number is 2 , the number of teeth of the rigid gear is 202, the number of teeth of the flexible gear is 200 , so the equation (9) may be in $i_{g}$ approximated to 1, and because one of flexible wheel and rigid wheel is fixed, so $d$ 'should represent the movement of the pitch circle diameter of the gear, and the difference between the pitch circle radius and $r_{m}$ is small, so it can be used $r^{\prime}$ instead, i.e $r_{m}=r^{\prime}=0.5 d^{\prime}$.

Therefore harmonic gear reducer motion error is determined to be approximately:

$$
\Delta \varphi_{h g}= \pm \frac{k_{B}}{\sqrt{z_{m p}}}\left[0.2\left(\sum_{1}^{j} \Delta F_{\Sigma j}+\frac{\pi d^{\prime}}{8 \omega_{0} i_{h}} \sum_{1}^{n} \Delta \rho_{n}\right)+0.3 \sqrt{\sum_{1}^{j}\left(\Delta F_{\Sigma j}\right)^{2}+\left(\frac{\pi d^{\prime}}{8 \omega_{0} i_{h}}\right)^{2} \sum_{1}^{n}\left(\Delta \rho_{n}\right)^{2}}\right] \frac{412.8}{d^{\prime}}
$$

For bi-directional transmission of harmonic gear, consider the impact of backlash, then

$$
\Delta \varphi_{h g}= \pm \frac{k_{B}}{\sqrt{z_{m p}}}\left[0.25\left(\sum_{1}^{j} \Delta F_{\Sigma j}+\frac{\pi d^{\prime}}{8 \omega_{0} i_{h}} \sum_{1}^{n} \Delta \rho_{n}\right)+0.4 \sqrt{\sum_{1}^{j}\left(\Delta F_{\Sigma j}\right)^{2}+\left(\frac{\pi d^{\prime}}{8 \omega_{0} i_{h}}\right)^{2} \sum_{1}^{n}\left(\Delta \rho_{n}\right)^{2}}\right] \frac{412.8}{d^{\prime}}
$$

Considering the structural rigidity, increased external load torque caused by the error, the total integrated error

$$
\Delta \varphi_{z h}= \pm \frac{k_{B}}{\sqrt{z_{m p}}}\left[0.25\left(\sum_{1}^{j} \Delta F_{\Sigma j}+\frac{\pi d^{\prime}}{8 \omega_{0} i_{h}} \sum_{1}^{n} \Delta \rho_{n}\right)+0.4 \sqrt{\sum_{1}^{j}\left(\Delta F_{\Sigma j}\right)^{2}+\left(\frac{\pi d^{\prime}}{8 \omega_{0} i_{h}}\right)^{2} \sum_{1}^{n}\left(\Delta \rho_{n}\right)^{2}}\right] \frac{412.8}{d^{\prime}}+\Delta_{e}
$$

In the type: $\Delta_{e}$ is external load torque error caused by the elastic shaft deformation due to flexible wheel.

\section{Theoretical Spectrum of Motion Error of Harmonic Reducer}

According to above error analysis of the harmonic reducer, MATLAB is used for spectrum analysis of its motion errors through each component of the error driven by the harmonic reducer. It is found that motion error of harmonic drive occurs in two basic frequency domain, namely $\omega t \sim 2 \omega t$ of the low-frequency domain and $2 i_{h} \omega t \sim k_{1} 2 i_{h} \omega t$ of the high-frequency domain; cam error is close to the teeth of the frequency of occurrence.

In the double-wave transmission, when the cam rotation a circle, flexible gear will rotate two teeth, namely the output shaft $\left(1 / i_{h}\right)$ circle. due to the polygonal cam and the impact of flexible bearing rollers error in cam rotation, forced Flexspline produce torsional deformation, and impact 
on the motion error. The polygonal degree and a flexible bearing rollers error cam torsional deflection of flexspline as show figure 2.

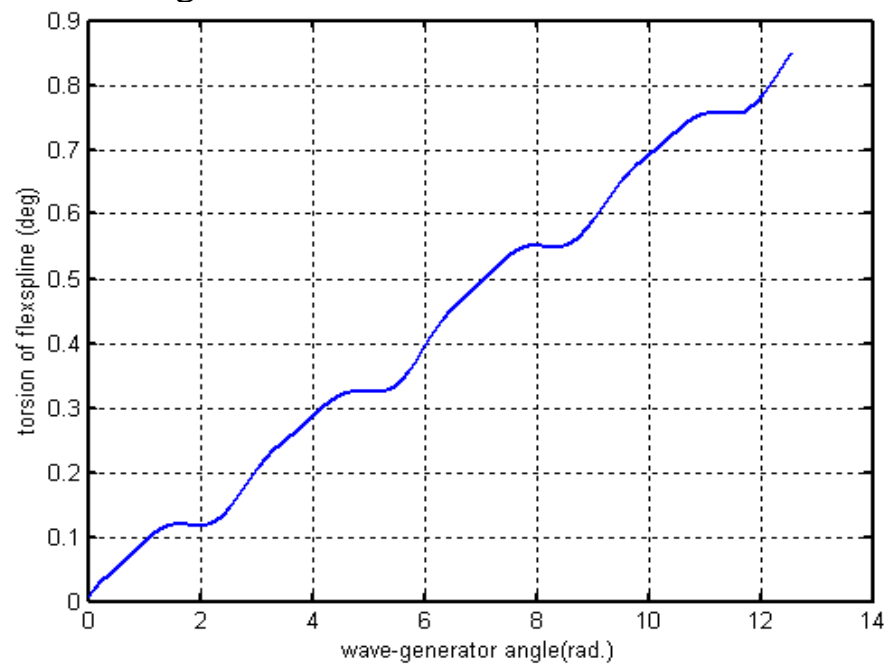

Fig. 2 The shaft torsional deformation to change map of flexible gear The motion error pooled analysis of each component, as shown in Table 1.

Tab. 1 Each component of the error and the corresponding frequency

\begin{tabular}{l|c|c}
\hline \multirow{2}{*}{ Corresponding frequency } & component of the error \\
\hline \multirow{2}{*}{ the low-frequency domain } & $\boldsymbol{\omega}$ & $\sum_{1}^{j} F_{r j}, E_{r b}$ \\
\cline { 2 - 3 } & $2 \omega t$ & $\sum_{1}^{j} \varphi_{\Sigma j}$ \\
\hline \multirow{2}{*}{ the high-frequency domain } & $2 i_{h} \omega t$ & $\sum_{1}^{j} \Delta \rho_{f \Sigma n}, f_{p b j}$ \\
\cline { 2 - 3 } & $k_{1} 2 i_{h} \omega t$ & $\sum_{1}^{j} \Delta \rho_{p \Sigma n}$ \\
\hline
\end{tabular}

In summary, it theoretically can be drawn that motion error curve and the differential motion error curve of harmonic reducer shown in Figure 3 and Figure 4.

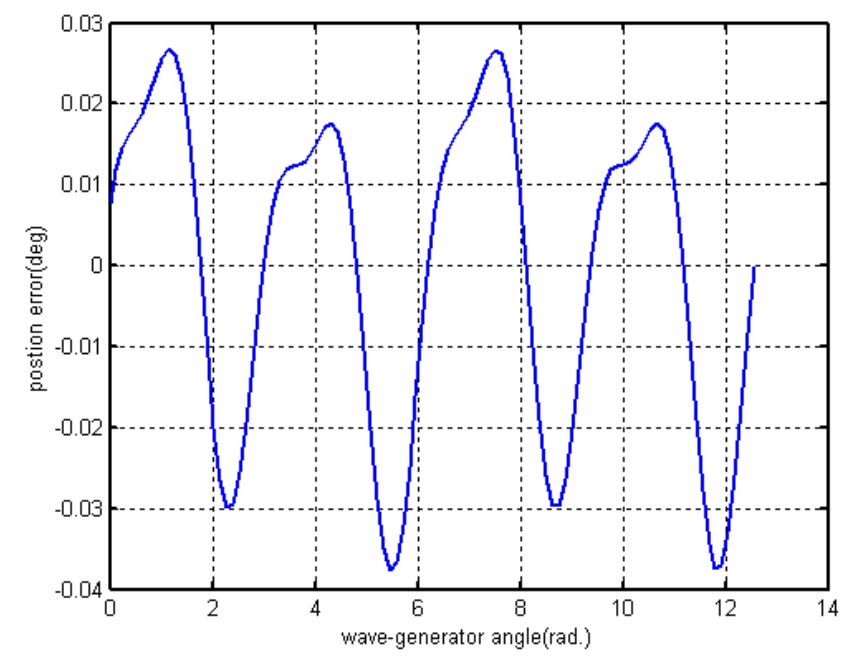

Fig. 3 motion error curve

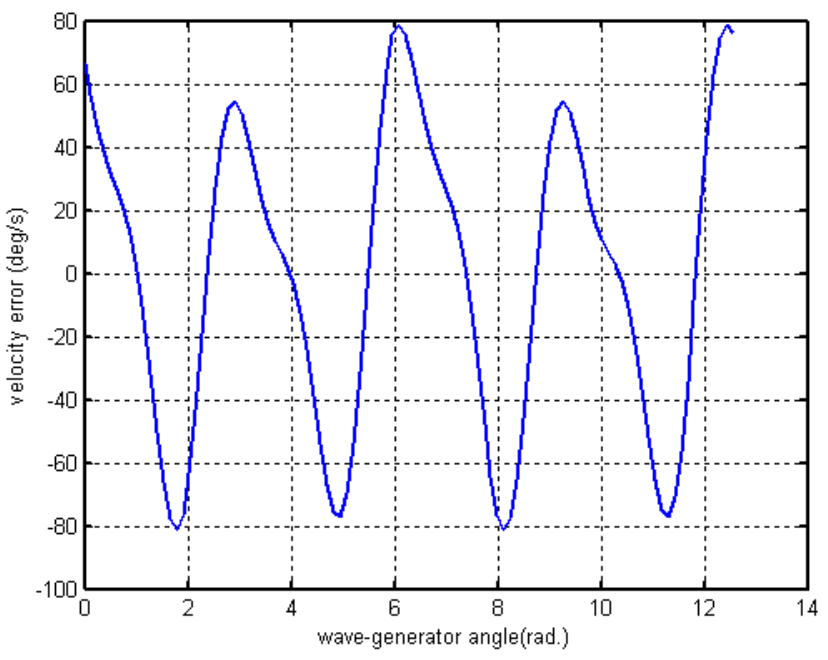

Fig. 4 motion error differential curve 


\section{Conclusion}

A comprehensive model of the driving error of harmonic reducer is theoretically developed and concluded, moreover its spectrum analysis is accomplished. So the theory basic is provided in order to establish the process quality control for ensuring the driving error of harmonic reducer to meet its technology requirements.

\section{Acknowledgement}

In this paper, the research was sponsored by the Research Project of Guangxi Institute of Education (Project No. 201012MS245) and the Research Project of Guangxi Technological College of Machinery and Electricity (Project No. [2010]KY16).

\section{References}

[1] An GU, Zhengxun LIU, Study on the Mechanism of High Accuracy Servo System [J], Mechatronics, 2002, (2): 28-29. (in Chinese)

[2] H. D. Taghirad, P. R. Belanger, An Experimental Study on Modeling and Identification of Harmonic Drive Systems [C], Proceedings of the 35th IEEE. Conference on Decision and Control, 1996: 4725 4730.

[3] Timothy D. Tuttle, Warren Seering, Modeling a Harmonic Drive Gear Transmission [J], IEEE. 1050-4729, 1993: 624 629.

[4] Mingrui HUANG, Zhili XU, Harmonic Gear Transmission Principle and Technology [J], Machinery Manufacturing, 1994, (3): 9 11. (in Chinese)

[5] Hongbing XIN, Harmonic Drive Technology and Its Research Trend [J], Journal of Beijing Institute of Light Industry, 1999, 17 (1): 30 35. (in Chinese)

[6] Yunwen SHEN, Qingtai YE, The Theory and Design of Harmonic Gear Drive[M], Beijing: Mechanical Industry Press, 1985: 212 235. (in Chinese)

[7] Harmonic Gear Reducer Test Method [A], Aerospace Industry Standard of the People's Republic of China, QJ1820-89, 1989: 1-5. (in Chinese)

[8] Di WANG, The Research on a New Type of the Harmonic Gear Reducer [D], Harbin: Harbin Institute of Technology, 2011: 24-35. (in Chinese)

[9] M. H. Ivanov, The Harmonic Gear Drive [M], Beijing: National Defense Industry Press, 1987: 23-28. (in Chinese) 